\begin{tabular}{|c|l|}
\hline Title & Electronic properties of metal-induced gap states formed at al kali-halide/metal interfaces \\
\hline Author(s) & Kiguchi, Manabu; Y oshikawa, Genki; Ikeda, Susumu; Saiki, Koichiro \\
\hline Citation & $\begin{array}{l}\text { Physical Review B, 71(15), 153401 } \\
\text { https://doi.org/10.1103/PhysRevB.71.153401 }\end{array}$ \\
\hline Issue Date & 2005-04.06 \\
\hline Doc URL & http://hdl.handle.net/2115/29747 \\
\hline Rights & Copyright $\odot 2005$ A merican Physical Society \\
\hline Type & article \\
\hline File Information & PRB71-15.pdf \\
\hline
\end{tabular}

Instructions for use 


\title{
Electronic properties of metal-induced gap states formed at alkali-halide/metal interfaces
}

\author{
Manabu Kiguchi, ${ }^{1, *}$ Genki Yoshikawa, ${ }^{2}$ Susumu Ikeda, ${ }^{2}$ and Koichiro Saiki ${ }^{1,2}$ \\ ${ }^{1}$ Department of Complexity Science \& Engineering, Graduate School of Frontier Sciences, The University of Tokyo, Hongo, \\ Bunkyo-ku, Tokyo 113-0033, Japan \\ ${ }^{2}$ Department of Chemistry, The University of Tokyo, Hongo, Bunkyo-ku, Tokyo 113-0033, Japan \\ (Received 21 September 2004; revised manuscript received 18 January 2005; published 6 April 2005)
}

\begin{abstract}
The spatial distribution and site distribution of metal-induced gap states (MIGS) are studied by thicknessdependent near-edge x-ray absorption fine structure (NEXAFS) and by comparing the cation and anion-edge NEXAFS. The thickness-dependent NEXAFS shows that the decay length of MIGS depends on an alkalihalide rather than a metal, and it is larger for alkali-halides with smaller band gap energies. By comparing the $\mathrm{Cl}$-edge and $\mathrm{K}$-edge NEXAFS for $\mathrm{KCl} / \mathrm{Cu}(001)$, MIGS are found to be states localizing at anion sites.
\end{abstract}

DOI: 10.1103/PhysRevB.71.153401

PACS number(s): 73.20.-r, 73.40.Ns

The electronic structure of semiconductor/metal or insulator/metal interfaces has attracted wide attention in relation to intriguing phenomena, such as band-gap narrowing, insulator-metal transition, and excitonic superconductivity. ${ }^{1-3}$ One of the characteristic electronic structures at the interfaces is the formation of a metal-induced gap state (MIGS). At the semiconductor (or insulator)/metal interface, a freeelectron-like metal wave function penetrates into a semiconductor (or insulator) side, and thus, an interface electronic state (MIGS) is formed in the band gap. ${ }^{4}$ The MIGS has been considered to determine the Schottky barrier height at the semiconductor/metal interface. ${ }^{5}$

While the decay length of the MIGS is an order of a few angstroms for typical semiconductors, it has generally been believed that the decay length of the MIGS into the insulator is negligible. This view is based on quite a plausible tightbinding model calculation. ${ }^{6}$ For a typical ionic insulator such as $\mathrm{LiCl}$ with a band gap of $9.4 \mathrm{eV}$, the decay length is estimated to be smaller than $0.1 \mathrm{~nm}$, which suggests that MIGS should be irrelevant at the insulator/metal interface. However, we have obtained unambiguous evidence that the MIGS are formed at atomically well-defined $\mathrm{LiCl} /$ metal interfaces by measuring the near-edge $\mathrm{x}$-ray absorption fine structure (NEXAFS).$^{7-9}$ With a decreasing $\mathrm{LiCl}$ thickness, a well pronounced prepeak appears at the bulk-edge onset in NEXAFS, suggesting the formation of an interface state in the band gap. The results of electron spectroscopies (x-ray photoemission spectroscopy and Auger electron spectroscopy) and theoretical calculation indicate that the prepeak originates not from the simple chemical bond, but from the proximity of ionic material to metals. Furthermore, it has been revealed that the decay length of the MIGS is as large as $0.3 \mathrm{~nm}$ and that the MIGS have a $p_{z}$-like structure, extending their electron clouds along the surface normal direction (parallel to the $z$ axis).

Although we have reported the existence of MIGS at the $\mathrm{LiCl} /$ metal interface, there still remain points to be discussed. First, the decisive factor to determine the decay length of the MIGS is not clear at the alkali-halide/metal interface. What is the decisive factor: metal substrates, alkali-halides, or their combinations? Second, it is not clear whether the MIGS extend uniformly in the surface parallel direction or if they are localized at the anion or cation site.
In order to determine the decisive factor, we have measured the NEXAFS for $\mathrm{KCl} /$ metal and discussed the decay length of the MIGS at the alkali-halide/metal interface by comparing the previous $\mathrm{LiCl} /$ metal results. To determine the site distribution of the MIGS, we have studied K-edge and Cl-edge NEXAFS for $\mathrm{KCl} /$ metal systems. The NEXAFS qualitatively provides information on the unoccupied $p$ electronic density of states of the X-ray absorbing atom. By comparing the intensity of the MIGS peak in K-edge and Cl-edge NEXAFS for $\mathrm{KCl} /$ metal, the density of states of the MIGS at the $\mathrm{K}$ and $\mathrm{Cl}$ sites are evaluated. Therefore, we can discuss whether the MIGS extend uniformly in the surface parallel direction or if they are localized at the anion or cation site. Finally we comment on the possibility of interface superconductivity.

The experiments were performed in a custom-designed ultrahigh-vacuum (UHV) system with a base pressure of 1 $\times 10^{-7} \mathrm{~Pa}$. Mechanically and electrochemically polished $\mathrm{Cu}(001)$ and $\mathrm{Ag}(001)$ crystals were cleaned by repeated cycles of Ar sputtering and annealing at $900 \mathrm{~K}$. $\mathrm{KCl}$ was evaporated from a Knudsen cell at the substrate temperature of $300 \mathrm{~K}$. The growth rate was monitored using a quartz crystal oscillator, and it was on the order of $1 \mathrm{ML}(0.31 \mathrm{~nm}) / \mathrm{min}$. Real-time observation of the crystallinity of the $\mathrm{KCl}$ films was done by reflection high-energy electron diffraction (RHEED). Sharp streaks were observed in the RHEED patterns of the grown $\mathrm{KCl}$ films, indicating that an epitaxial $\mathrm{KCl}(001)$ film grew in a layer-by-layer fashion. ${ }^{7,8}$

$\mathrm{Cl}-K$-edge and $\mathrm{K}$ - $K$-edge NEXAFS measurements were carried out at the soft x-ray double-crystal monochromator station BL-11B of the Photon Factory in the High Energy Accelerator Research Organization. ${ }^{10}$ The energy resolution of the $\mathrm{Ge}(111)$ monochromator was about $1.5 \mathrm{eV}$. The fluorescence-yield detection method was employed to obtain NEXAFS data by using an UHV-compatible gas-flow proportional counter with $\mathrm{P} 10$ gas $\left(10 \% \mathrm{CH}_{4}\right.$ in $\left.\mathrm{Ar}\right)$ as a detector. The $\mathrm{Cl} 1 s$ x-ray photoemission spectrum (XPS) was measured using X-ray synchrotron radiation at $2900 \mathrm{eV}$ with a RIGAKU XPS-7000 concentric hemispherical analyzer. In the XPS for $\mathrm{KCl} /$ metal systems, the width of the $\mathrm{Cl} 1 s$ peak does not change with the film thickness, nor does a satellite 


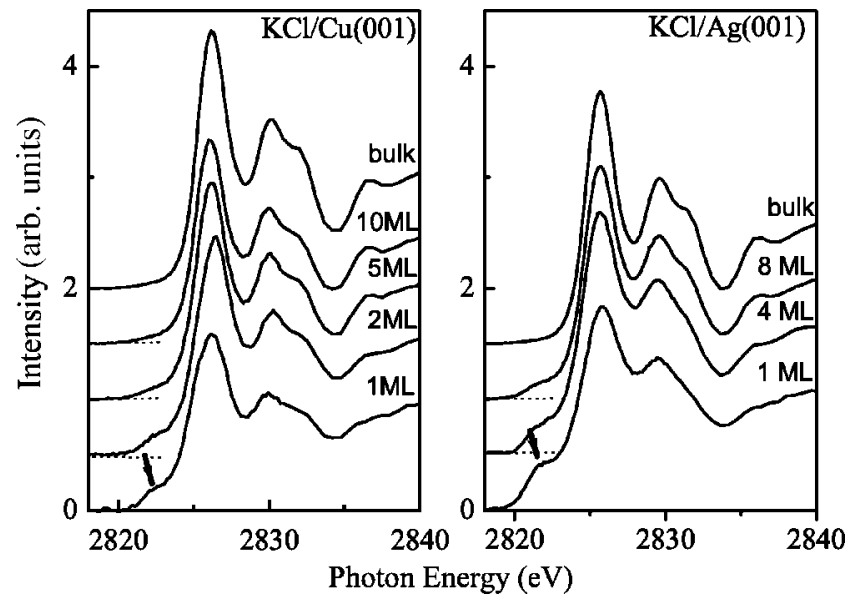

FIG. 1. The Cl- $K$-edge NEXAFS spectra of $\mathrm{KCl}$ films grown on $\mathrm{Cu}(001)$ and $\mathrm{Ag}(001)$ for various thicknesses of the $\mathrm{KCl}$ layer taken at the x-ray incidence angle of $15^{\circ}$. All of the spectra are normalized by their edge jumps.

peak appear, indicating that a chemical bond $(\mathrm{Cl}-\mathrm{Cu}$ or $\mathrm{Cl}-\mathrm{Ag}$ bonds) is not formed at the $\mathrm{KCl} /$ metal interface.

Figure 1 shows the $\mathrm{Cl}-K$-edge NEXAFS for the epitaxially grown $\mathrm{KCl}$ film on $\mathrm{Ag}(001)$ and $\mathrm{Cu}(001)$ taking at grazing $\mathrm{X}$-ray incidence $\left(15^{\circ}\right)$ for various thicknesses of the $\mathrm{KCl}$ layer. All the spectra are normalized by their edge jumps. In the thin film region, a well pronounced prepeak, which originates from the MIGS, appears just below the bulk-edge onset. In the following, we will discuss the spatial distribution of the MIGS at the alkali-halide/metal interface by analyzing the intensity of this prepeak.

First, the spatial distribution of the MIGS in the surface normal direction is discussed in terms of the decay length of the MIGS. Figure 2 shows the intensity of the prepeak (not normalized by the edge jump unlike in Fig. 1) as a function of the film thickness. Here, we assume that the probing depth of the NEXAFS is much greater (typically $\geqslant 1000 \mathrm{~nm}$ ) than the atomic scale, and the intensity of the MIGS $[f(x)]$ at the distance $x$ from the interface can be represented as $I_{0} \exp (-x / \lambda)$, where $I_{0}, x$, and $\lambda$ are the intensity of the MIGS at the interface and decay length, respectively. The intensity of the MIGS $[F(X)$; film thickness $X]$ observed by

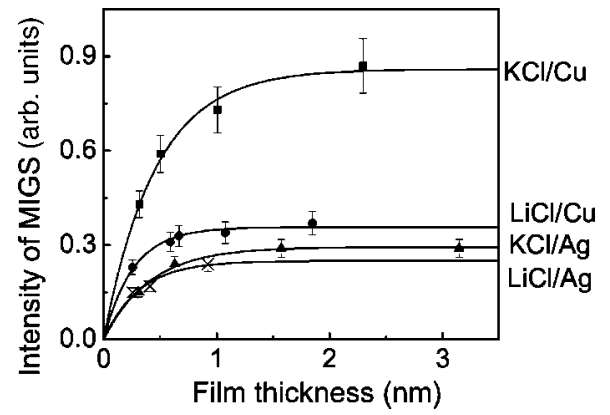

FIG. 2. The intensity of the prepeak (not normalized by the edge jump, unlike in Fig. 1) versus the film thickness, where the curves indicate the results of the least-square fit to $F(X)$. The intensity of the prepeak for the alkali-halide film was obtained by subtracting the bulk component from the spectra.

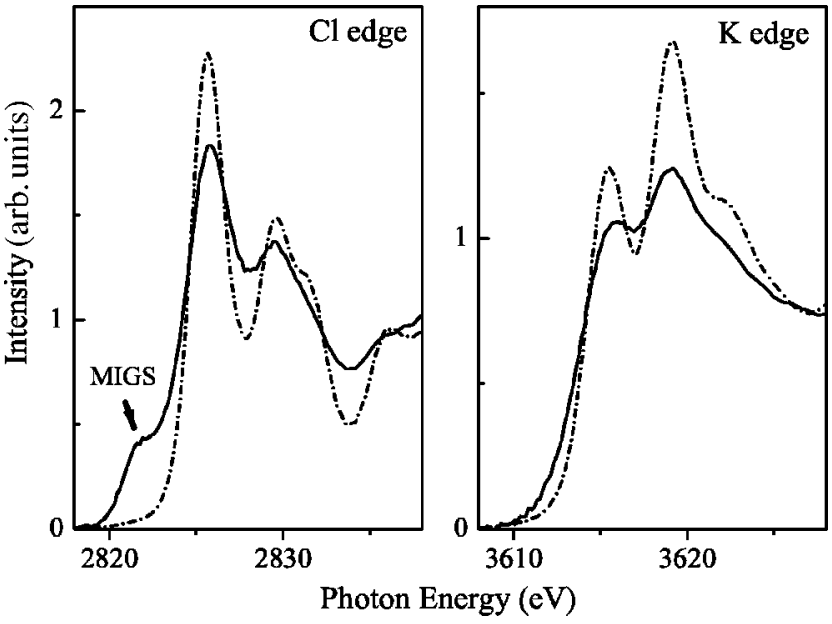

FIG. 3. The $\mathrm{Cl}-K$-edge and $\mathrm{K}-K$-edge NEXAFS spectra of 1-ML-thick $\mathrm{KCl}$ films grown on $\mathrm{Cu}(001)$ taken at the $\mathrm{x}$-ray incidence angle of $15^{\circ}$ (line), together with the spectra of the bulk $\mathrm{KCl}$ (dotted line).

the NEXAFS is thus represented as $I_{0} \lambda[1-\exp (-X / \lambda)]$ by integrating $f(x)$ from 0 to $X$. By fitting the experimental data with $F(X)$, the decay length is determined to be $0.46 \mathrm{~nm}$ for $\mathrm{KCl} / \mathrm{Cu}(001), 0.41 \mathrm{~nm}$ for $\mathrm{KCl} / \mathrm{Ag}(001), 0.26 \mathrm{~nm}$ for $\mathrm{LiCl} / \mathrm{Cu}(001)$, and $0.29 \mathrm{~nm}$ for $\mathrm{LiCl} / \mathrm{Ag}(001)$. The result for the fitting is included in Fig. 2.

The intensity of the MIGS at the interface is 19 (a.u.) for $\mathrm{KCl} / \mathrm{Cu}(001), 7.1$ for $\mathrm{KCl} / \mathrm{Ag}(001), 14$ for $\mathrm{LiCl} / \mathrm{Cu}(001)$, and 8.6 for $\mathrm{LiCl} / \mathrm{Ag}(001)$. It can be found that the decay length of the MIGS depends on the kind of alkali-halide, not on the metal. This conclusion is consistent with the recent theoretical calculation conducted by Arita et al. ${ }^{11}$ They have evaluated the decay length of the MIGS for various alkalihalide/metal combinations by the $a b$ initio calculation, in which they conclude that the decay length of the MIGS is close to half the lattice constant of the alkali-halides. Since the lattice constant of $\mathrm{KCl}(L=0.63 \mathrm{~nm})$ is larger than that of $\mathrm{LiCl}(L=0.51 \mathrm{~nm})$, the present experimental results are consistent with the theoretical calculation results.

In contrast with the decay length, the intensity of the MIGS at the interface depends on the metal substrate. The intensity of the MIGS at the alkali-halide/ $\mathrm{Cu}(001)$ interface $\left(I_{0}\right)$ is larger than that at the alkali-halide/ $\mathrm{Ag}(001)$ interface. This difference can be explained by the density of states of metal substrates near the Fermi energy. Since the penetration of electrons in the metal causes the MIGS, the intensity of the MIGS at the interface relates closely with the density of states at the Fermi energy, $E_{F}$. The density of states at $E_{F}$ for $\mathrm{Cu}$ is larger than that for $\mathrm{Ag}\left(r_{s}\right.$ parameter: 2.7 for $\mathrm{Cu}, 3.0$ for $\mathrm{Ag})$. It is thus reasonable that the intensity of the MIGS at the alkali-halide/ $\mathrm{Cu}(001)$ interface is larger than that at the alkali-halide/ $\operatorname{Ag}(001)$ interface.

In the previous section, we have discussed the spatial distribution of the MIGS in the surface normal direction. We now discuss the spatial distribution of the MIGS in the surface parallel direction. The $\mathrm{Cl}-K$-edge and $\mathrm{K}-K$-edge NEXAFS provide information on the unoccupied $\mathrm{Cl}-p$ and $\mathrm{K}-p$ electronic densities of states. The topmost surface of the 

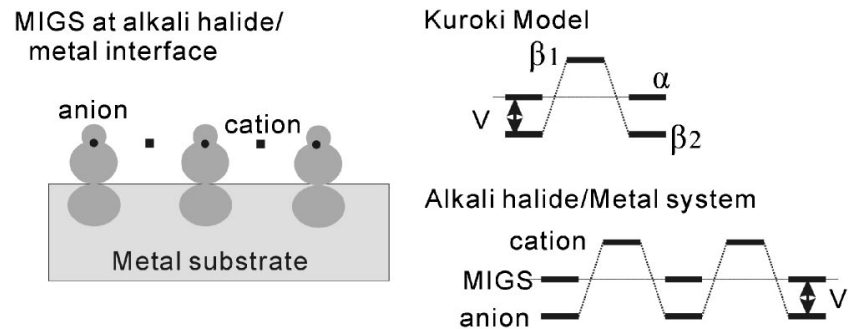

FIG. 4. Systematic view of the MIGS at the alkali-halide/metal interface. The positions of the cation (box) and the anion (circle) are shown in the figure. (Right) Kuroki model and a multiband model of alkali-halide/metal interfaces. In the Kuroki model, the carrier band $(\alpha)$ interacts repulsively with the insulating band $\left(\beta_{2}\right)$. In the alkali-halide/metal system, the MIGS band interacts with the anion band.

alkali-halide/face-centered-cubic (fcc) metal heterostructures is just the (001) face of the alkali-halide crystal, consisting of the same number of cations and anions. Therefore, we can discuss the site distribution of the MIGS in the surface parallel direction by comparing the intensity of the MIGS peak in both $\mathrm{Cl}$-edge and $\mathrm{K}$-edge NEXAFS for the $\mathrm{KCl} /$ metal system. Figure 3 shows $\mathrm{Cl}$ - $K$-edge and $\mathrm{K}-K$-edge NEXAFS for the 1-ML-thick $\mathrm{KCl}$ film on $\mathrm{Cu}(001)$. The prepeak originating from the MIGS is clearly observed at the $\mathrm{Cl}$ edge, while the prepeak is not observed at the $\mathrm{K}$ edge, indicating that the MIGS are formed only at the anion site. On the other hand, the polarization-dependent NEXAFS results show that the MIGS are the states extending their electron clouds along the surface normal direction. ${ }^{9}$ Considering these NEXAFS results and our previous calculation results (Fig. 3 in Ref. 9), we can present a systematic view of the spatial distribution of the MIGS for the alkali-halide/metal interface as seen in Fig. 4.

The localization of the MIGS at the anion site can be discussed by considering the calculation results done by Arita et al. ${ }^{11}$ Generally, the MIGS can be divided into two groups: one having a conduction-band character and the other having a valence-band character at the insulator side of the interface. Noguera and Bordier introduced the energy $E_{Z C P}$ which categorizes the character of the MIGS. ${ }^{6}$ According to their theory, an electronic state has a conduction-band character when its electronic energy is larger than $E_{Z C P}$, and vice versa. On the other hand, the charge transfer between the insulator and the metal is determined by the energy position of $E_{Z C P}$ relative to the Fermi energy $\left(E_{F}\right)$. The charge is transferred from the insulator to the metal, if $E_{Z C P}>E_{F}$. Arita et al. evaluate the charge transfer for various alkalihalide/metal combinations by ab initio calculations, and find that the charge transfer from the insulator to the metal commonly occurs for the alkali-halide/metal systems. ${ }^{11}$ This indicates that $E_{Z C P}$ is higher than $E_{F}$ for the alkali-halide/metal systems, and thus, the electronic state near $E_{F}$ has a valence band character. Since the MIGS are electronic states formed near $E_{F}$, the MIGS have a valence band character, and they are localized at the anion site.

Finally, let us point out that the electronic structure specific to the alkali-halide/metal interfaces can have implications for superconductivity. In the previous study, we propose a possibility of the MIGS working favorably for exciton-mediated superconductivity. ${ }^{9,11}$ In the presence of the MIGS at the alkali-halide/metal interface, an exciton [associated with the wide-band gap of the alkali-halide] and carriers (from the MIGS) coexist within one atomic distance, a situation which is favorable for strong interaction between the carriers and the exciton. This strong interaction leads to a possible ground for superconductivity by the exciton mechanism proposed by Ginzburg et al. ${ }^{3}$

The localization of the MIGS at the anion sites provides another possibility of superconductivity proposed by Kuroki and Aoki. ${ }^{12}$ They propose that repulsively interacting systems consisting of a carrier band and an insulating band can become superconducting, and the system can be effectively mapped to an attractive Hubbard model. In their model, the metallic band is assumed to interact only with the anion sites (or the cation sites). Figure 4 shows the Kuroki model, where the carrier band $(\alpha)$ interacts repulsively $(V)$ with the insulating band $\left(\beta_{2}\right)$. At the alkali-halide/metal interface, the MIGS have their amplitudes only on the anion sites, so that the MIGS should interact primarily with the anion sites, which just corresponds to the situation considered in Kuroki model. The $\beta_{1}, \beta_{2}$, and $\alpha$ bands in the Kuroki model correspond to the anion, cation, and MIGS bands in the alkalihalide/metal systems, as shown in Fig. 4. So we may envisage that the alkali-halide/metal interface may provide a possible ground for superconductivity, although quantitative prediction for $T_{c}$ could not be done at the present stage.

This work was supported by a Grant-in-Aid for Scientific Research and Special Coordination Fund from the Ministry of Education of Japan. (Creative Scientific Research Project, Grant No. 14GS0207). The present work was performed under the approval of the Photon Factory Program Advisory Committee (Grant No. PF-PAC No.2001G336).

\footnotetext{
*Present address: Department of Chemistry, Hokkaido University, Sapporo 060-0810, Japan.

${ }^{1} \mathrm{P}$. W. Anderson in Elementary Excitation in Solids, Molecules and Atoms, Part A, edited by J. A. Devreese et al. (Plenum Press, New York, 1974)

${ }^{2}$ R. Arita, Y. Tanida, K. Kuroki, and H. Aoki, Phys. Rev. B 64, 245112 (2001).
}

\footnotetext{
${ }^{3}$ High-Temperature Superconductivity, edited by V. L. Ginzburg and D. A. Kirzhnits (Consultants Bureau, New York, 1982).

${ }^{4}$ S. G. Louie and M. L. Cohen, Phys. Rev. B 13, 2461 (1976).

${ }^{5}$ J. Tersoff, Phys. Rev. Lett. 52, 465 (1984).

${ }^{6}$ C. Noguera and G. Bordier, J. Phys. III 4, 1851 (1994).

${ }^{7}$ M. Kiguchi, K. Saiki, T. Sasaki, Y. Iwasawa, and A. Koma, Phys. Rev. B 63, 205418 (2001).
} 
${ }^{8}$ M. Kiguchi, S. Entani, K. Saiki, H. Inoue, and A. Koma, Phys. Rev. B 66, 155424 (2002).

${ }^{9}$ M. Kiguchi, R. Arita, G. Yoshikawa, Y. Tanida, M. Katayama, K. Saiki, A. Koma, and H. Aoki, Phys. Rev. Lett. 90196803 (2003).
${ }^{10}$ T. Ohta, P. M. Stefan, M. Nomura and H. Sekiyama, Nucl. Instrum. Methods Phys. Res. A 246, 373 (1986).

${ }^{11}$ R. Arita, Y. Tanida, K. Kuroki, and H. Aoki, Phys. Rev. B 69 115424 (2004).

${ }^{12}$ K. Kuroki and H. Aoki, Phys. Rev. Lett. 693820 (1992). 